\title{
Warped Wigner-Hough Transform for Defect Reflection Enhancement in Ultrasonic Guided Wave Monitoring
}

\author{
Luca De Marchi, ${ }^{1}$ Emanuele Baravelli, ${ }^{1}$ Giampaolo Cera, ${ }^{1}$ \\ Nicolò Speciale, ${ }^{1}$ and Alessandro Marzani $^{2}$ \\ ${ }^{1}$ Department of Electronics, Information and Computer Systems (DEIS), University of Bologna, \\ 40136 Bologna, Italy \\ ${ }^{2}$ Department of Civil, Environmental and Materials Engineering (DICAM), University of Bologna, \\ 40136 Bologna, Italy
}

Correspondence should be addressed to Alessandro Marzani, alessandro.marzani@unibo.it

Received 27 August 2012; Revised 19 October 2012; Accepted 20 October 2012

Academic Editor: Zhongqing Su

Copyright (C) 2012 Luca De Marchi et al. This is an open access article distributed under the Creative Commons Attribution License, which permits unrestricted use, distribution, and reproduction in any medium, provided the original work is properly cited.

\begin{abstract}
To improve the defect detectability of Lamb wave inspection systems, the application of nonlinear signal processing was investigated. The approach is based on a Warped Frequency Transform (WFT) to compensate the dispersive behavior of ultrasonic guided waves, followed by a Wigner-Ville time-frequency analysis and the Hough Transform to further improve localization accuracy. As a result, an automatic detection procedure to locate defect-induced reflections was demonstrated and successfully tested by analyzing numerically simulated Lamb waves propagating in an aluminum plate. The proposed method is suitable for defect detection and can be easily implemented for real-world structural health monitoring applications.
\end{abstract}

\section{Introduction}

In recent years, ultrasonic guided waves (GWs) have received a great deal of attention among nondestructive tests community due mainly to the ability to travel long distances without substantial attenuation and to employ multimode/-frequency examination for defect classification and sizing. Among the various techniques based on GWs, the detection of defects in plates-like structures by means of Lamb waves has been, and is still, widely investigated [1-6] due to the variety of potential applications. Since the propagation characteristics are directly related to both the inherent structure and mechanical properties of the medium, the dispersiveness of GWs can reveal important information for structural 


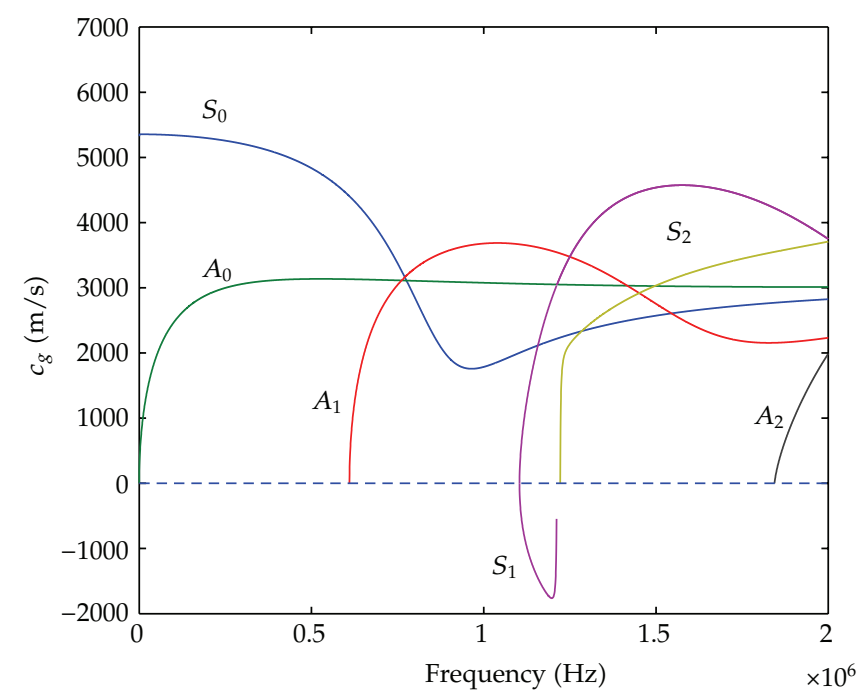

Figure 1: Group velocity dispersion curves for the Lamb waves propagating in a $2.54 \mathrm{~mm}$ thick aluminum plate (Young modulus $E=69 \mathrm{GPa}$, Poisson's coefficient $v=0.33$, and density $\rho=2700 \mathrm{~kg} / \mathrm{m}^{3}$ ).

health monitoring purposes. Unfortunately, several different modes appear simultaneously in the signal. These modes overlap in both time and frequency domains, and simple Fourier analysis techniques are not able to separate them.

Thus, identification of Lamb modes is a challenging step in the process of damage detection. However, recent works in the area of time-frequency representations (TFRs) $[7,8]$ show great potential for applications in nondestructive evaluation and material characterization. Specifically, TFRs can provide an effective tool for the interpretation of GWs, whose multimodal and dispersive nature make them an extremely complicated class of ultrasonic signals.

This work proposes a time-frequency (TF) energy density function approach that makes use of known dispersion characteristics for a propagating wave mode in order to compensate for the effect of dispersion and locate defects in plate-like structures. Our approach will be illustrated through a relevant case study, in which defects are to be located on an aluminum plate where Lamb waves are excited.

\section{Numerical Simulation of Lamb Wave Propagation}

Let us consider an aluminum plate of thickness is $h=2.54 \mathrm{~mm}$, Young's modulus $E=69 \mathrm{GPa}$, and Poisson's ratio $v=0.33$. The proposed processing requires the computation of the group velocity dispersion curves for the plate. For such task, today several formulations and tools are available. For instance, in uniform waveguides the group velocity $c_{g}(f)$ can be estimated by means of analytical-based formulations [9], semianalytical finite element (SAFE) simulations [10], and by using standard finite element codes [11]. Recent developments allow the computation of the dispersion curves also in the case of irregular waveguides [12]. The results shown in Figure 1 were obtained by a using free-SAFE-based tool that can be downloaded at http://www.guiguw.com/ [10]. 

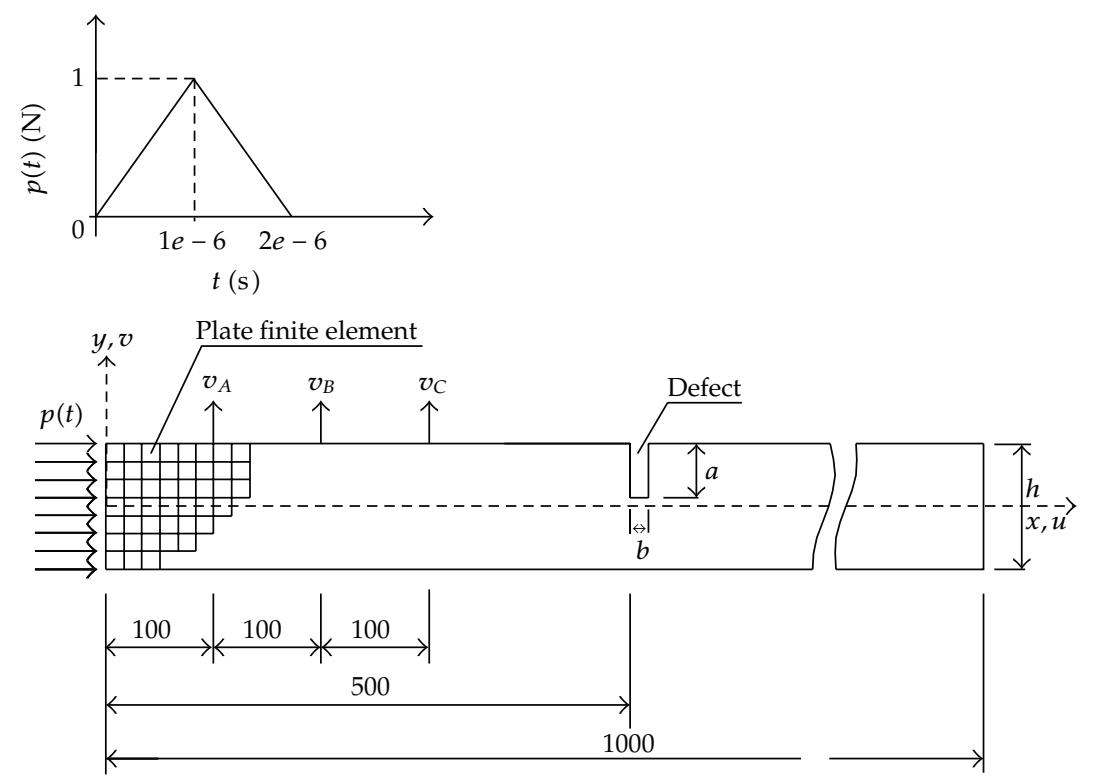

Figure 2: Schematic representation of the damaged aluminum plate used in the time-transient FEM simulations (plate dimensions are in $\mathrm{mm}$ ). The spatial distribution and time-amplitude shape of the actuation pulse is also shown.

As a second step, time waveforms related to Lamb waves propagating in the aluminum plate were obtained numerically by means of dedicated Finite Element (FEM) simulations using Abaqus explicit [13]. Thanks to the Lamb problem symmetry, a $x-y$ plane strain condition was assumed, as shown in Figure 2. A notch of width $b=0.25 \mathrm{~mm}$ and depth $a$ was considered, such that $a / h=0.3$. The assumed notch location was on the top side at the center of the plate $(x=500 \mathrm{~mm})$. Lamb waves were excited by applying an impulsive force $p(t)$ to the left edge of the plate towards the positive $x$-direction: this mainly stimulates the symmetric $S_{0}$ mode. The force was shaped in time as a triangular window with a total duration of $2 \mu \mathrm{s}$ (see the top left of Figure 2) in order to excite consistent Lamb waves up to $500 \mathrm{kHz}$.

For instance, a similar excitation was obtained in [14] by focusing, through conventional optics, a laser beam to a straight line acting along the plate edge. It is shown in such work that the $S_{0}$ mode can be excited by means of such experimental setup.

To ensure accuracy to the time-transient finite element simulations [15], the plate domain was discretized with elements of maximum side length $L_{\max }=0.125 \mathrm{~mm}$, and the time integration step was kept $\Delta t<1 e-8 \mathrm{sec}$.

Time-dependent out-of-plane displacements $v(t)$ were recorded at three points on the top side of the plate $(y=h / 2)$, namely, $A, B$, and $C$, respectively, located at $x_{A}=100 \mathrm{~mm}$, $x_{B}=200 \mathrm{~mm}$, and $x_{C}=300 \mathrm{~mm}$. The recorded waveforms are shown in Figure 3 . The leftmost peak in each signal corresponds to the passage of the excited $S_{0}$ mode through the recording position (path 1, in Figure 4), while oscillations in the central part of the waveforms are due to defect-induced reflections (path 2, in Figure 4), which also excite the slower $A_{0}$ mode. Spreading of these oscillations clearly reveals the effect of dispersion. Finally, further reflections from the plate edges (path 3, in Figure 4) are responsible for the complicated behaviour observed in the rightmost part of the signals. 


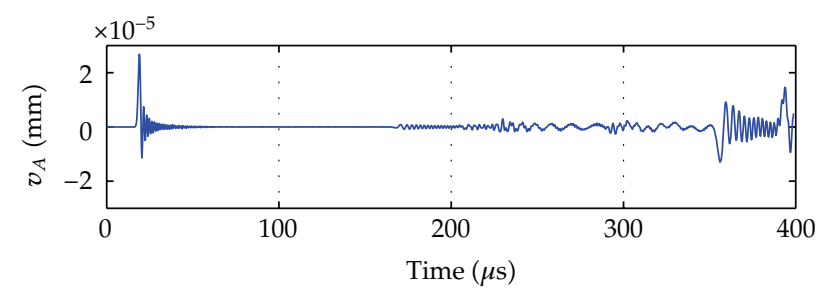

(a)

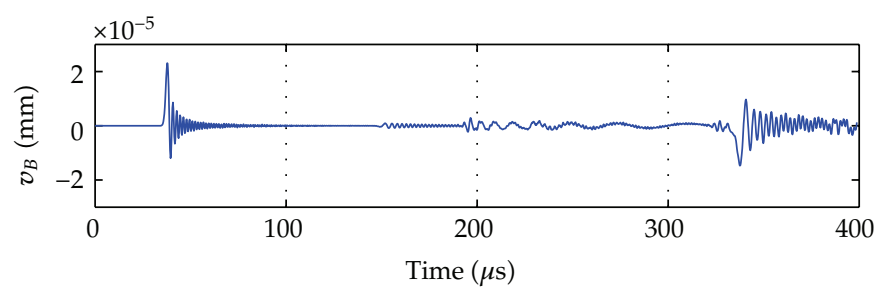

(b)

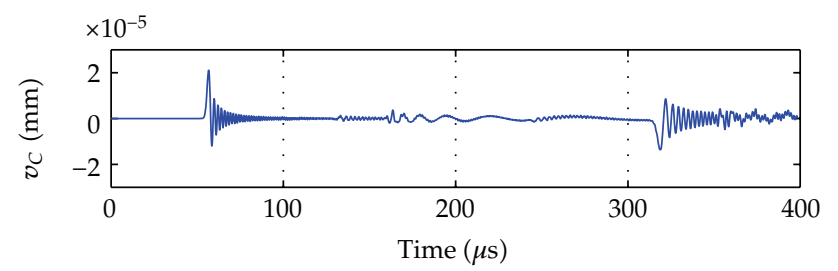

(c)

Figure 3: Time-dependent out-of-plane displacements recorded on the top side $(y=h / 2)$ of the plate at (a) $x_{A}=100 \mathrm{~mm}$, (b) $x_{B}=200 \mathrm{~mm}$, and (c) $x_{C}=300 \mathrm{~mm}$.

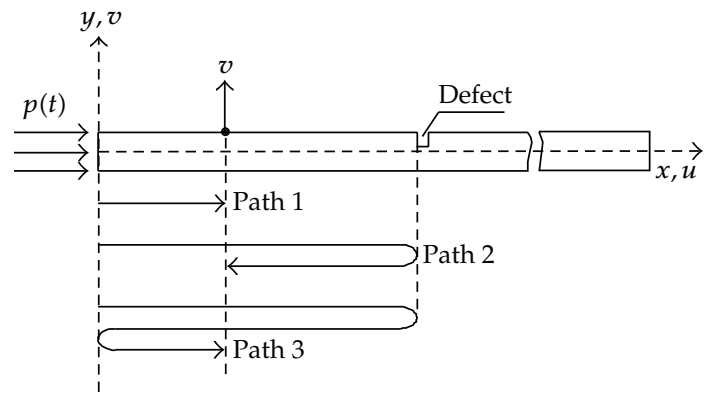

Figure 4: Schematic representation of the multiple paths traveled by the waves detected in a given acquisition point.

\section{Mathematical Tools}

The defect location procedure can be divided in three steps: (i) Warped Frequency Transform to remove the dispersive behaviour of the $S_{0}$ mode; (ii) equalization procedure to enhance weak reflections; (iii) Wigner-Hough Transform to distinguish $S_{0}$ reflections from other interfering waves. Such steps are detailed in the following subsections. 


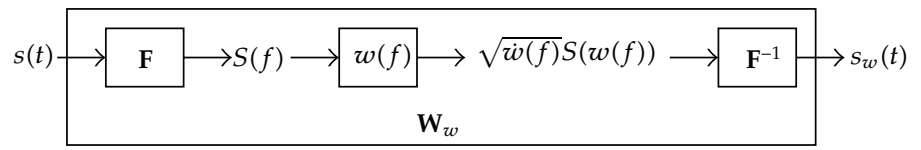

Figure 5: Computational flow of the frequency warping operator $\mathbf{W}_{w} \cdot \mathbf{F}$ and $\mathbf{F}^{-1}$ are the direct and inverse Fourier Transform operators, respectively, while $w(f)$ and $\dot{w}(f)$ are the warping map and its first derivative.

\subsection{The Warped Frequency Transform (WFT)}

The WFT is a unitary time-frequency transformation that produces a flexible sampling of the time-frequency domain [16].

Given a generic signal $s(t)$ whose Fourier Transform (FT) is $S(f)$, the frequency warping operator $\mathbf{W}_{w}$ transforms the original waveform into a warped version $s_{w}(t)$ by reshaping the frequency domain through a properly designed warping map $w(f)$. The procedure is depicted in Figure 5.

The WFT can be used to compensate dispersion in GWs [17] by defining the warping map through the following relationship:

$$
K \frac{d w^{-1}(f)}{d f}=\frac{1}{c_{g}(f)}
$$

Equation (3.1) relates the functional inverse $w^{-1}(f)$ of the map to the inverse of the group velocity curve, that is, $1 / c_{g}(f)$, of the wave that we want to consider. $K$ is a normalization parameter selected so that $w^{-1}(0.5)=w(0.5)=0.5$. As shown in Figure 1, for acoustic emission below $500 \mathrm{kHz}$, only the two fundamental waves $A_{0}$ and $S_{0}$ can propagate through the plate. It is assumed that mostly $S_{0}$ is actuated, therefore the group velocity curve of $S_{0}$ has been used to build $w(f)$.

If $s(t)$ is an undamped guided wave at a traveled distance $D$ from the actuator, its FT is given by $S(f)=S_{0}(f) \cdot e^{-j 2 \pi \int_{0}^{f} \tau(\alpha) d \alpha}$ where $S_{0}(f)$ is the FT of the actuated wave (incipient pulse) and $\tau(f)=D / c_{g}(f)$ represents the frequency-dependent group delay of $S_{0}$ in this case. Using (3.1), $S(f)$ can be rewritten as

$$
\begin{aligned}
S(f) & =S_{0}(f) \cdot e^{-j 2 \pi D \int_{0}^{f}\left(1 / c_{g}(\alpha)\right) d \alpha} \\
& =S_{0}(f) \cdot e^{-j 2 \pi w^{-1}(f) K D} .
\end{aligned}
$$

By applying warping and exploiting the invertibility property of the map, that is, $w^{-1}[w(f)]=f$, yields to a signal $s_{w}(t)$ whose spectrum is

$$
\mathbf{F W}_{w}\{S(t)\}=\left[\sqrt{\dot{w}(f)} S_{0}(w(f))\right] \cdot e^{-j 2 \pi f K D}
$$

The linear dependence on the warped frequency in this equation shows that the dispersive effect is converted in a simple warped time-delay $(K D)$ proportional to the distance. Dispersion is therefore compensated, and the resulting signal $s_{w}$ can be equivalently plotted 
as a function of the distance from the source, thus allowing to locate defects of the excited waveguide by detecting the corresponding reflected waves.

It is worth noticing that, despite the different formalism, the processing described above is substantially analogous to the ones presented in $[18,19]$. However, in many practical applications such dispersion compensation is not sufficient to ensure a reliable estimation of wave traveled distance, due to the weakness of reflections and to the interfering presence of different modes. For these reasons, necessary further processing steps are introduced in this work, as will be shown in the following sections.

\subsection{Wave Equalization}

By means of the warping procedure described in the previous section, a realignment of the time-frequency content of $S_{0}$ waves in vertical lines is produced. However, in general the energy of the waves scattered by defects is much lower than the energy of the incident wave, especially for small defects. To overcome this problem, the energy of incident and reflected waves in the acquired signal can be conveniently equalized. Such task can be accomplished with a simple but effective procedure based on a local averaging of the acquired signal. Indicating with $L A\left(\left|s_{w}(x)\right|\right)$ the local average of $\left|s_{w}(x)\right|$ in the neighborhood of $x$, the equalized signal $s_{w e}(x)$ is obtained as

$$
s_{w e}(x)=\frac{s_{w}(x)}{\max \left\{L A\left(\left|s_{w}(x)\right|\right), T\right\}}
$$

where $T$ is a given threshold, set as the $5 \%$ of the maximum value of $\left|s_{w}(x)\right|$, which is used to avoid the undesired amplification of numerical noise when the signal is absent. In experimental data, the value of $T$ must be set according to the SNR of the acquisition setup.

The warped and equalized versions of the signal in Figure 3(a) are plotted in Figures $6(\mathrm{a})$ and $6(\mathrm{c})$, respectively. The equalization factor $\max \left\{L A\left(\left|s_{w}(x)\right|\right), T\right\}$ is depicted in Figure 6(b).

\subsection{The Wigner-Hough Transform (WHT)}

After equalization, defect detection can be performed automatically with a further processing of the signal $s_{w e}(x)$. It is worth noticing that the energy of spurious contribution (caused by multimodal propagation or mode conversion) is quite high in the equalized signal compared to the energy of compensated $S_{0}$ waves. In the example of Figure 6(c), the peak related to the $S_{0}$ wave reflected by the defect (indicated as $S_{0}$ path 2) is just about twice as high as the peak in the following mode-converted wave $\left(A_{0}\right)$. Therefore it is quite difficult to implement simple thresholding procedures capable of distinguishing different wave modes in this representation.

However, defect-induced reflections of the analyzed mode ( $S_{0}$ in this example) appear in the compensated waveform as well-localized spikes, thus producing vertical maxima lines in a TF representation, whose (warped) time location can be directly converted to the defect position. On the contrary, spurious contributions related to different modes $\left(A_{0}\right.$ in this example) show a peculiar frequency modulation due to a different group velocity curve from the one of $S_{0}$. This can be clearly observed in a simple TFR of $s_{w e}(t)$, provided by the shorttime Fourier transform (STFT), shown in Figure 7. 


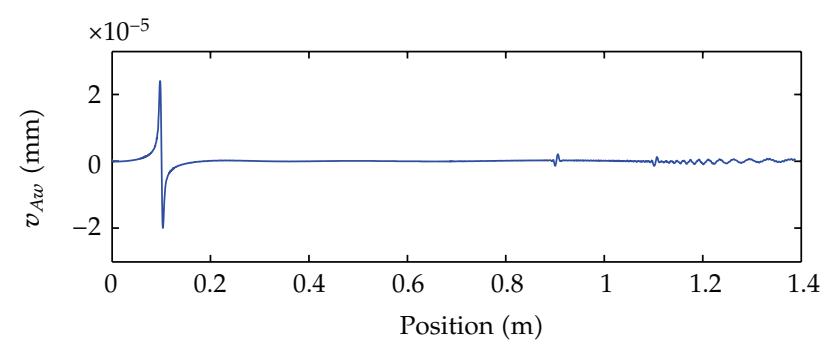

(a) Warped signal

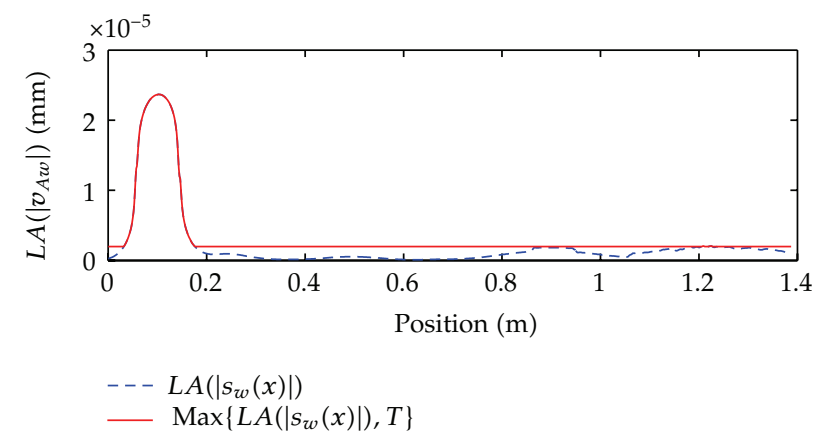

(b) Equalizing signal

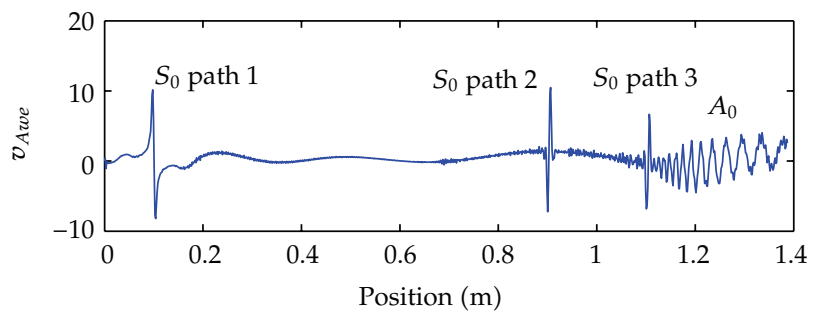

(c) Equalized warped signal

Figure 6: (a) Warped version $v_{A w}$ of the signal $v_{A}$ represented in Figure 3(a). (b) Equalization factor for the same signal. (c) Equalized warped signal $v_{A w e}$ as a result of the equalization procedure.

Other energy distributions, such as the Wigner-Ville distribution (WVD), defined as

$$
W_{s_{w e}}(t, f)=\int_{-\infty}^{+\infty} s_{w e}\left(t+\frac{\tau}{2}\right) s_{w}^{*}\left(t-\frac{\tau}{2}\right) \cdot e^{-j 2 \pi f \tau} d \tau
$$

can be used to further improve the effectiveness of the representation. In fact, WVD provides optimal energy localization of linear chirp signals in the time-frequency plane [20]. It follows that applying vertical line detection algorithms to the WVD of the compensated signals provides an asymptotically optimal detector of wave propagating distances.

In particular, automatical detection of the desired lines of energy maxima can be performed by applying the Hough Transform (HT) [21] to the WVD, resulting in the so-called Wigner-Hough Transform (WHT) [22]. Generally speaking, the HT is an image processing tool that performs an integration on all the possible lines of a given image $I$ and maps the value of each integral to a $(\rho, \theta)$ plane corresponding to the polar parametrization of 


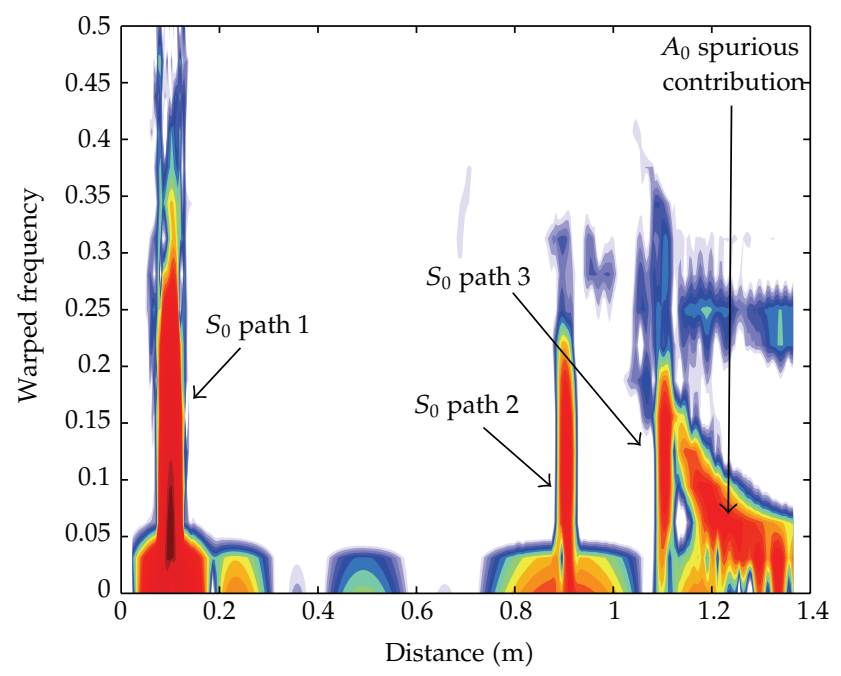

Figure 7: Spectrogram of the equalized waveform $v_{\text {Awe }}$ represented in Figure 6(c).

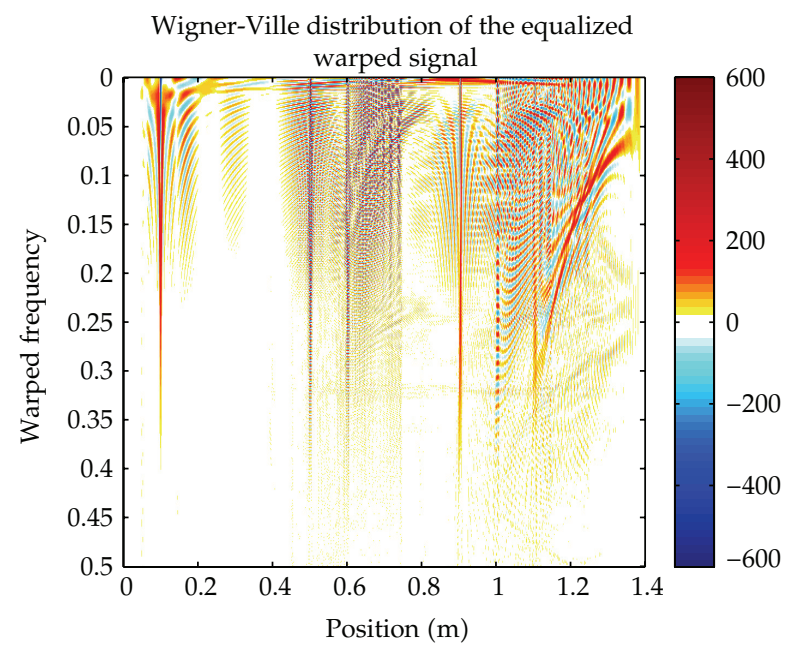

Figure 8: Wigner-Ville distribution (WVD) of the equalized waveform $v_{\text {Awe }}$ represented in Figure 6(c).

the lines. High-intensity pixels concentrated on straight lines on $I$ will therefore produce peaks in the $(\rho, \theta)$ domain. In the Wigner-Hough Transform, the input image corresponds to the WVD of the considered signal and, in our approach, emphasis is placed in finding vertical lines, located at $\theta=\{\pi / 2,3 \pi / 2\}$. Therefore, in the WHT, the portion which corresponds to these angles is isolated, and the $\rho$ value corresponding to detected peaks represents the distance traveled by the wave. One of the major limitations of the WVD is the presence of interference terms between different spectral components of the analyzed waveform induced by the WVD. However, it is worth noticing that this inconvenience is largely compensated through the integration performed by the Hough operator, as these undesired components appear as alternating positive peaks and negative valleys in the TF plane. 


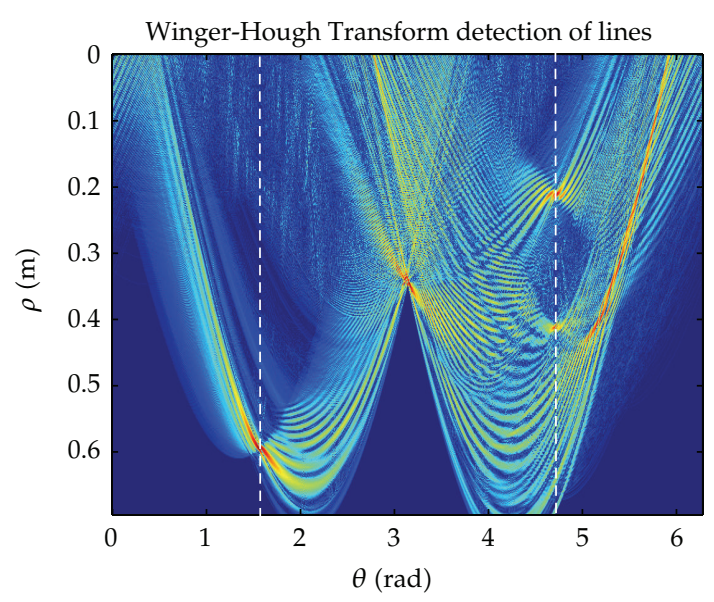

Figure 9: Hough Transform of the Wigner-Ville distribution (WHT) represented in Figure 8. Peaks at $\theta=$ $\{\pi / 2,3 \pi / 2\}$ (dashed lines) correspond to vertical lines in Figure 7; $\rho$ coordinates provide the distance from the center of the analyzed image.

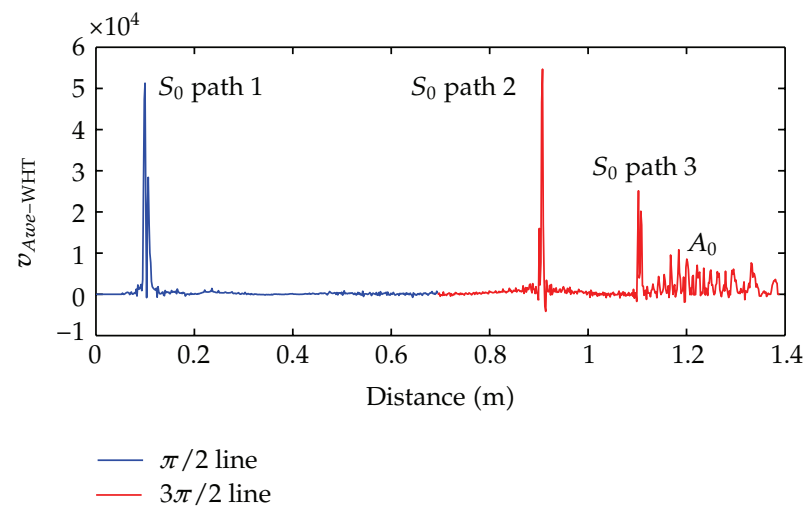

Figure 10: $v_{\text {Awe-WHT }}$ extracted for the values of $\theta=\pi / 2$ and $3 \pi / 2$ from the WHT of Figure 9 , computed for the signal $v_{\text {Awe }}$ represented in Figure 8.

\section{Procedure of the Method}

The mathematical tools detailed in the previous section can be efficiently implemented by the following processing steps.

(i) The discrete WFT can be computed with the approach described in [23]. In essence, the warped signal is obtained by performing a nonuniform Fourier Transform [24] followed by an inverse Fourier Transform. Fast-Fourier algorithms can be exploited to compute both the direct nonuniform and the inverse transforms.

(ii) In the second step, warped signals are equalized in amplitude, according to (3.4). The local averaging window applied in the right hand term of the same equation is about $9 \mathrm{~cm}$ in length (it is worth recalling that, in the warped domain, time intervals are related to distances). 


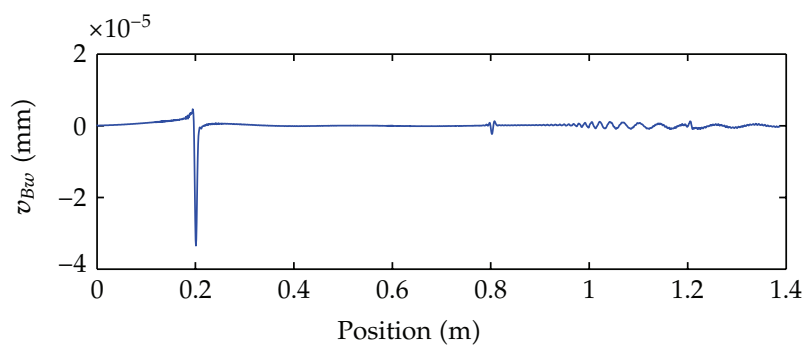

(a) Warped signal

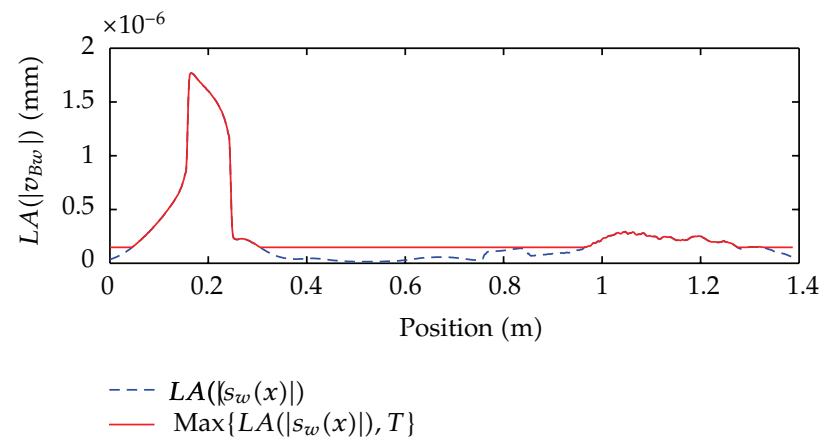

(b) Equalizing signal

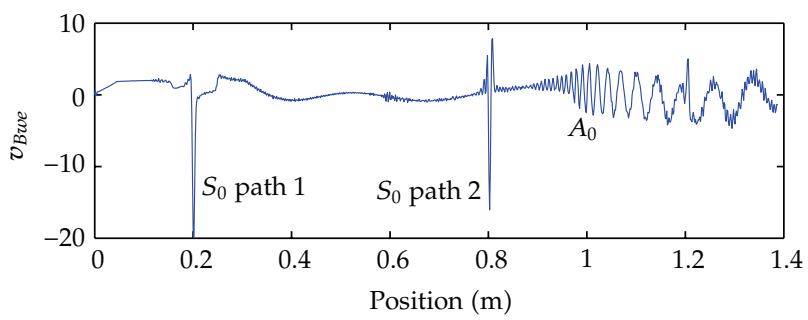

(c) Equalized warped signal

Figure 11: (a) Warped version $v_{B w}$ of the signal $v_{B}$ presented in Figure 3(b). (b) Equalization factor for the same signal. (c) Equalized warped signal $v_{B w e}$ as a result of the equalization procedure.

(iii) The software (Time-Frequency Toolbox-TFTB) which computes the WignerVille distribution adopted in this study is available for academic use at http://tftb.nongnu.org/. In the same tool, also the code for Hough Transform computation is provided. However, for wave propagating distance estimation, the calculation of the Hough Transform coefficients in the whole $(\rho, \theta)$ plane is redundant, as described in Section 3.3. For this reason, in our approach such calculation is simplified with a simple integration of WVD coefficients across frequencies.

\section{Numerical Results}

The WVD of the equalized warped signal in Figure 6 is depicted in Figure 8. As it can be seen, two vertical lines appears in correspondence to the actual traveled distances of the incident 


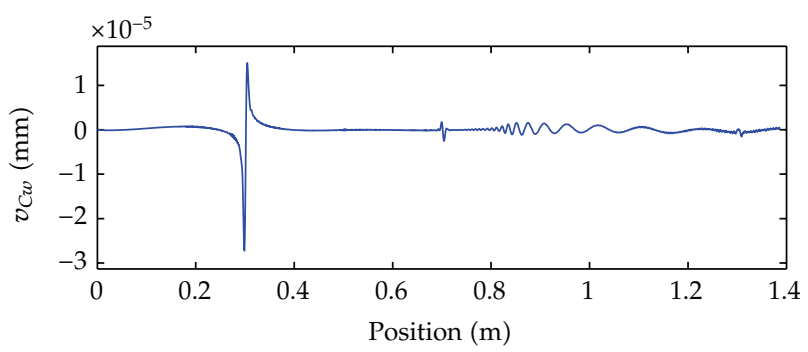

(a) Warped signal

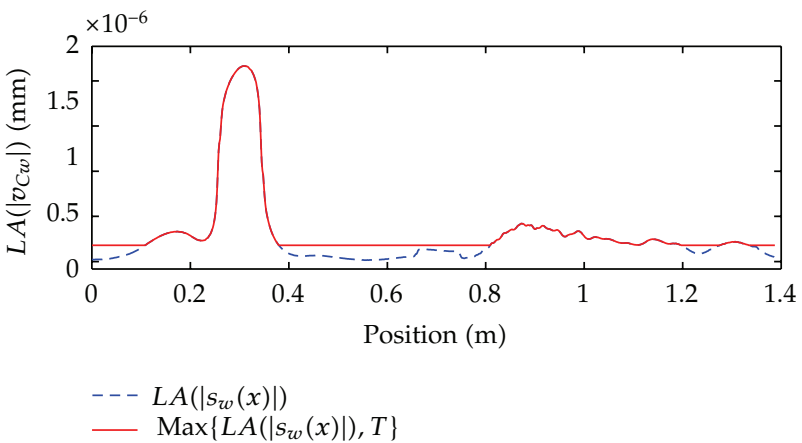

(b) Equalizing signal

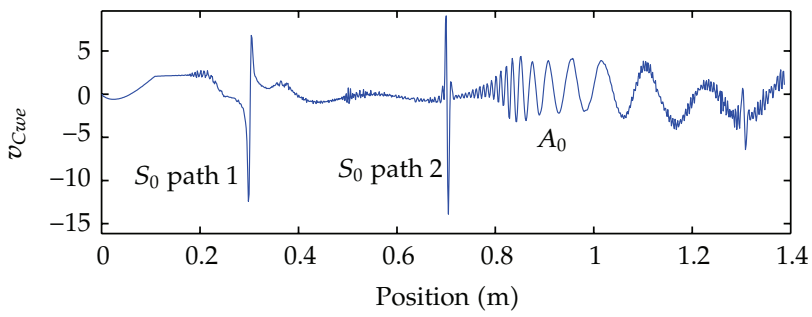

(c) Equalized warped signal

Figure 12: (a) Warped version $v_{C w}$ of the signal $v_{C}$ presented in Figure 3(c). (b) Equalization factor for the same signal. (c) Equalized warped signal $v_{C w e}$ as a result of the equalization procedure.

and scattered wave (at 0.1 and $0.9 \mathrm{~m}$, resp.) together with spurious contribution due to different modes of propagation and interference terms.

The computation of the WHT, that is, the Hough Transform on the WVD, produces the image depicted in Figure 9, where three peaks can be associated with $\theta=\pi / 2$ and $3 \pi / 2$. The peaks ordinates represent the difference between the effective distance of propagation of a given wave and a reference distance of propagation of $0.7 \mathrm{~m}$, which corresponds to the half of the maximum considered propagation distance in the analyses of $1.4 \mathrm{~m}$.

By extracting the values related to $\theta=\pi / 2$ and $3 \pi / 2$ in the WHT and reordering them according to the distance from the origin, a novel signal $s_{w e-W H T}$ is obtained. In such signal, depicted in Figure 10, the peaks related to the actual traveled distances of $S_{0}$ waves clearly emerge with respect to the spurious contribution due to mode-converted waves, thus greatly simplifying the definition of automated distance estimation procedures. In particular, the amplitude of the peak related to the reflected $S_{0}$ (path 2) is about 10 times higher than 


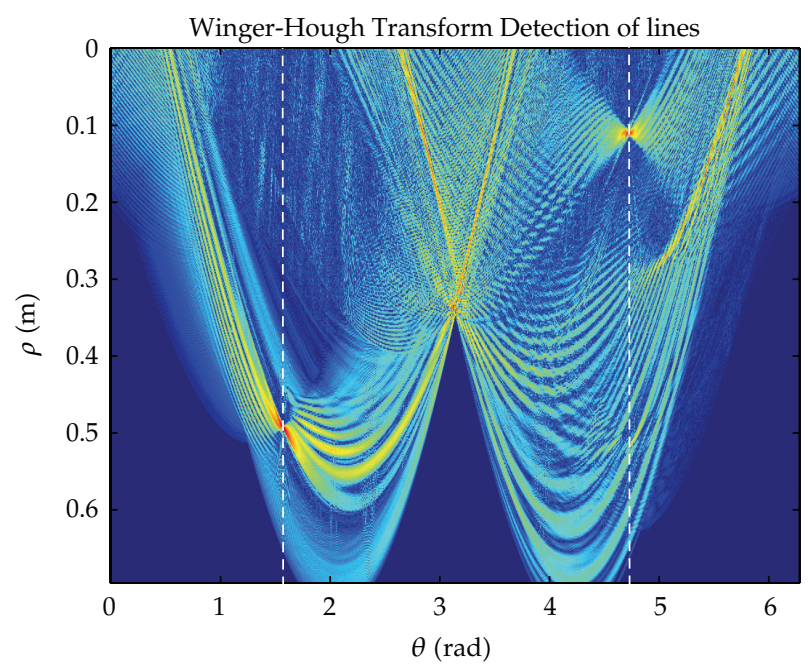

Figure 13: Wigner-Hough Transform (WHT) of the equalized warped signal $v_{B w e}$ represented in Figure 11(c). Peaks at $\theta=\{\pi / 2,3 \pi / 2\}$ (dashed lines) correspond to vertical lines in the Wigner-Ville distribution; $\rho$ coordinates provide the distance from the center of the analyzed domain.

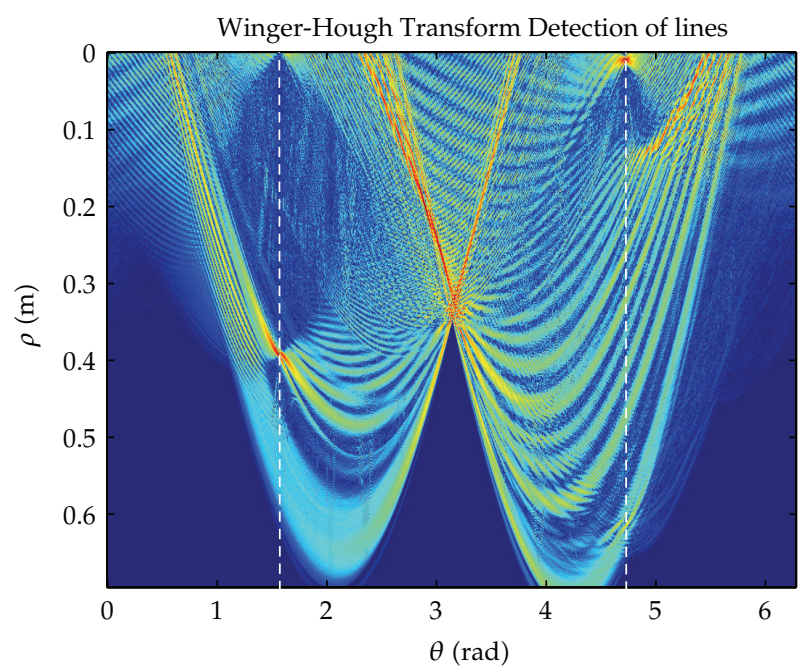

Figure 14: Wigner-Hough Transform of the equalized warped signal $v_{\text {Cwe }}$ represented in Figure 12(c). Peaks at $\theta=\{\pi / 2,3 \pi / 2\}$ (dashed lines) correspond to vertical lines in the Wigner Ville distribution; $\rho$ coordinates provide the distance from the center of the analyzed domain.

the maximum values of spurious contribution, that is, five times more with respect to the dispersion compensated wave of Figure 6 . Similar results have been obtained by processing the signals $v_{B}$ and $v_{C}$ represented in Figures 3(b) and 3(c).

Also for these signals, in fact, it can be seen that the path followed by the compensated mode $S_{0}$ can be tracked by observing local peaks in the warped signals, as illustrated in Figures 11(a) and 12(a), and the location of the defect can be inferred by the position of reflected peaks. However, the amplitude of the reflected $S_{0}$ peak, at $0.8 \mathrm{~m}$ in Figure 11(a) and $0.7 \mathrm{~m}$ in Figure 12(a), is much smaller compared to that on the incident $S_{0}$ mode and 


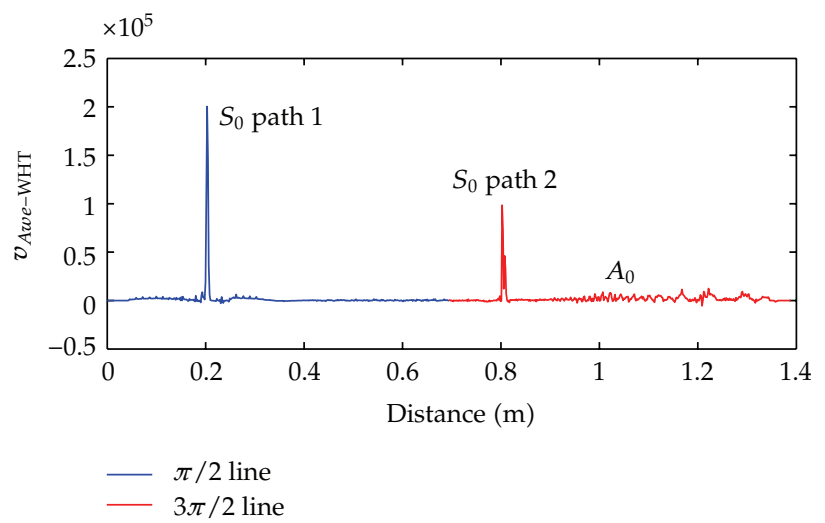

Figure 15: $v_{B w e-W H T}$ extracted for the values $\theta=\pi / 2$ and $3 \pi / 2$ from the WHT of $v_{B w e}$ represented in Figure 13.

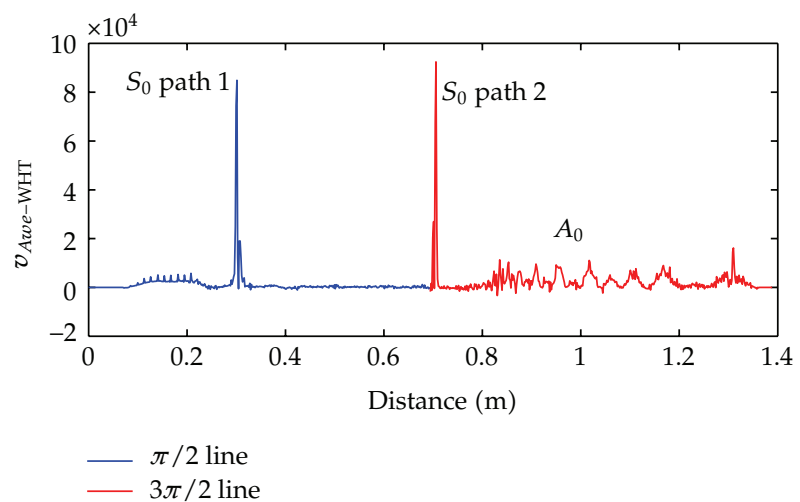

Figure 16: $v_{C w e-W H T}$ extracted for the values $\theta=\pi / 2$ and $3 \pi / 2$ from the WHT of $v_{C w e}$ represented in Figure 14 .

comparable to that of the reflected spurious $A_{0}$ wave. It follows that the location of defects by exploiting only warped signals could be difficult in real noisy applications. As for the first case discussed, the proposed robust and automated approach involves first an equalization of the warped signal to emphasize the amplitude of reflection-induced peaks and next the detection of vertical maxima lines in a space-frequency representation (WHT) of the equalized warped signals.

The Wigner-Hough Transform, in fact, appears as a suitable tool to isolate $S_{0}$ components and locate defect-induced reflections, as it can be seen form Figures 13 and 14, where the WHT of the equalized warped signals $v_{B w e}$ and $v_{C w e}$, respectively, are displayed.

Local maxima at $\theta=\pi / 2$ and $\theta=3 \pi / 2$ can be easily detected, and the corresponding $\rho$ coordinates provide the distance traveled by the incident and reflected $S_{0}$ waveform components, respectively.

From the extraction of peak coordinates in the waveforms $s_{w e-W H T}$ depicted in Figures 10,15 , and 16, the defect responsible for reflections is located at $x=503 \mathrm{~mm}, x=502 \mathrm{~mm}$, and $x=502 \mathrm{~mm}$, respectively. Errors with respect to the actual defect position $(x=500 \mathrm{~mm})$ are thus below $3 \mathrm{~mm}$, which roughly corresponds to the minimum wavelength associated with 
the excited Lamb waves. Similar good results were found by considering different defect depths and positions, not shown here for the sake of brevity.

\section{Conclusions}

The work described the application of a Warped Wigner-Ville analysis to improve defect detectability of conventional Lamb wave inspection systems. The proposed equalization approach effectively enhances the amplitude of relevant peaks in warped signals, where dispersion for a GW mode of interest has been removed. This procedure may encounter limitations in the presence of especially noisy signals, as spurious components might be erroneously amplified. However, several alternatives are possible, including more sophisticated preprocessing algorithms under investigation and the averaging of multiple acquisitions.

The Wigner-Ville distribution of the equalized signal is then computed. The presence of interference terms is largely compensated through the integration of the time-frequency decomposition performed by the Hough operator.

In the resulting Winger-Hough Transform representation, vertical lines associated with relevant acoustic events can be detected. This allows for the separation of overlapping Lamb waves. In particular, it was shown with numerical examples that the contribution of $S_{0}$ can be highlighted and the one due to interfering terms (such as $A_{0}$ wave) deeply attenuated.

Finally, with simple thresholding procedures, the information about the distance traveled by the incident and reflected components of a monitored wave can be easily recognized. Thanks to its very high precision the developed tool could pave the way for a new class of procedures to locate defects in waveguides.

\section{References}

[1] T. Ghosh, T. Kundu, and P. Karpur, "Efficient use of Lamb modes for detecting defects in large plates," Ultrasonics, vol. 36, no. 7, pp. 791-801, 1998.

[2] J. L. Rose, Ultrasonic Waves in Solid Media, Cambridge University Press, New York, NY, USA, 1999.

[3] Y. Lu, L. Ye, Z. Su, and C. Yang, "Quantitative assessment of through-thickness crack size based on Lamb wave scattering in aluminium plates," NDT and E International, vol. 41, no. 1, pp. 59-68, 2008.

[4] L. De Marchi, M. Ruzzene, B. Xu, E. Baravelli, and N. Speciale, "Warped basis pursuit for damage detection using Lamb waves," IEEE Transactions on Ultrasonics, Ferroelectrics, and Frequency Control, vol. 57, no. 12, pp. 2734-2741, 2010.

[5] E. Dehghan Niri and S. Salamone, "A probabilistic framework for acoustic emission source localization in plate-like structures," Smart Materials and Structures, vol. 21, no. 3, Article ID 035009, 2012.

[6] T. Kundu, H. Nakatani, and N. Takeda, "Acoustic source localization in anisotropic plates," Ultrasonics, vol. 52, no. 6, pp. 740-746, 2012.

[7] R. Benz, M. Niethammer, S. Hurlebaus, and L. J. Jacobs, "Localization of notches with Lamb waves," Journal of the Acoustical Society of America, vol. 114, no. 2, pp. 677-685, 2003.

[8] A. Leger and M. C. Deshamps, Ultrasonic Wave Propagation in Non Homogeneous Media, Springer, New York, NY, USA, 2009.

[9] M. J. S. Lowe, "Matrix techniques for modeling ultrasonic waves in multilayered media," IEEE Transactions on Ultrasonics, Ferroelectrics, and Frequency Control, vol. 42, no. 4, pp. 525-542, 1995.

[10] P. Bocchini, A. Marzani, and E. Viola, "Graphical user interface for guided acoustic waves," Journal of Computing in Civil Engineering, vol. 25, no. 3, pp. 202-210, 2011.

[11] F. Chen and P. D. Wilcox, "The effect of load on guided wave propagation," Ultrasonics, vol. 47, no. 1-4, pp. 111-122, 2007.

[12] L. De Marchi, A. Marzani, N. Speciale, and E. Viola, "Prediction of pulse dispersion in tapered waveguides," NDT and E International, vol. 43, no. 3, pp. 265-271, 2010. 
[13] “Abaqus-release 6. 12," 2012, http://www.simulia.com/.

[14] M. Sale, P. Rizzo, and A. Marzani, "Semi-analytical formulation for the guided waves-based reconstruction of elastic moduli," Mechanical Systems and Signal Processing, vol. 25, no. 6, pp. 22412256, 2011.

[15] I. Bartoli, F. Lanza Di Scalea, M. Fateh, and E. Viola, "Modeling guided wave propagation with application to the long-range defect detection in railroad tracks," NDT and E International, vol. 38, no. 5, pp. 325-334, 2005.

[16] S. Caporale, L. De Marchi, and N. Speciale, "Frequency warping biorthogonal frames," IEEE Transactions on Signal Processing, vol. 59, no. 6, pp. 2575-2584, 2011.

[17] L. De Marchi, A. Marzani, N. Speciale, and E. Viola, "A passive monitoring technique based on dispersion compensation to locate impacts in plate-like structures," Smart Materials and Structures, vol. 20, no. 3, Article ID 035021, 2011.

[18] R. Sicard, J. Goyette, and D. Zellouf, "A numerical dispersion compensation technique for time recompression of Lamb wave signals," Ultrasonics, vol. 40, no. 1-8, pp. 727-732, 2002.

[19] P. D. Wilcox, "A rapid signal processing technique to remove the effect of dispersion from guided wave signals," IEEE Transactions on Ultrasonics, Ferroelectrics, and Frequency Control, vol. 50, no. 4, pp. 419-427, 2003.

[20] E. Chassande-Mottin and P. Flandrin, "On the time-frequency detection of chirps1," Applied and Computational Harmonic Analysis, vol. 6, no. 2, pp. 252-281, 1999.

[21] L. Shapiro and G. Stockman, Computer Vision, Prentice Hall, New York, NY, USA, 2001.

[22] S. Barbarossa, "Analysis of multicomponent LFM signals by a combined Wigner-Hough transform," IEEE Transactions on Signal Processing, vol. 43, no. 6, pp. 1511-1515, 1995.

[23] S. Caporale, L. De Marchi, and N. Speciale, "Fast computation of frequency warping transforms," IEEE Transactions on Signal Processing, vol. 58, no. 3, pp. 1110-1121, 2010.

[24] L. Greengard and J.-Y. Lee, "Accelerating the nonuniform fast fourier transform," SIAM Review, vol. 46, no. 3, pp. 443-454, 2004. 


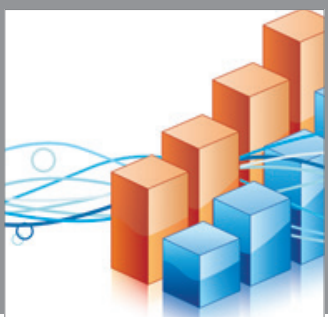

Advances in

Operations Research

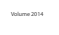

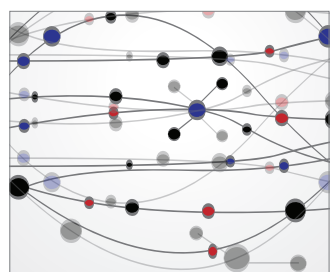

\section{The Scientific} World Journal
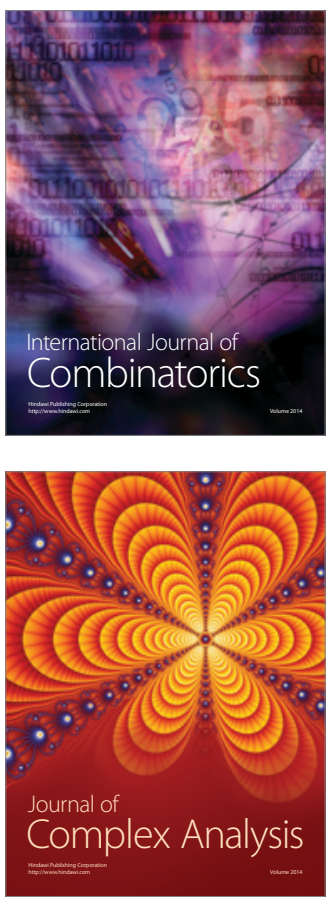

International Journal of

Mathematics and

Mathematical

Sciences
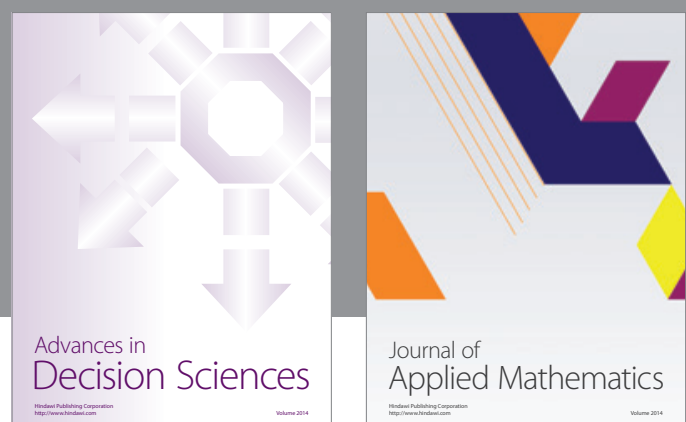

Journal of

Applied Mathematics
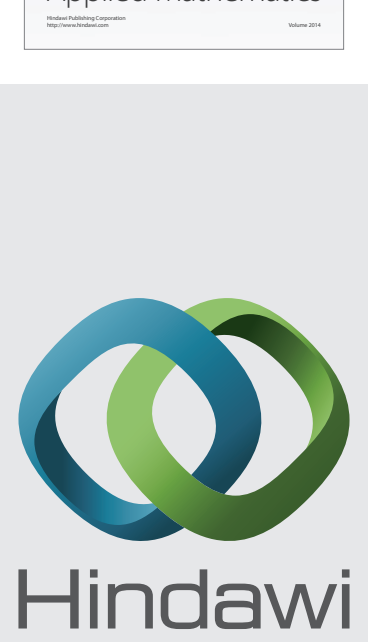

Submit your manuscripts at http://www.hindawi.com
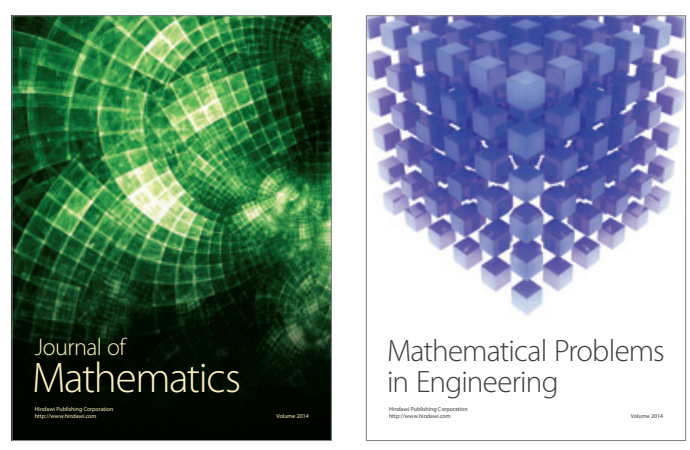

Mathematical Problems in Engineering
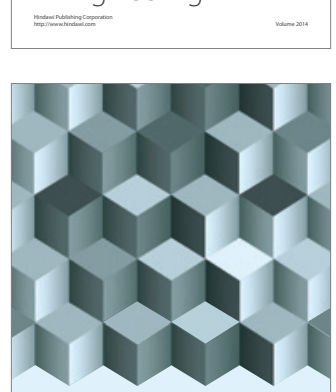

Journal of

Function Spaces
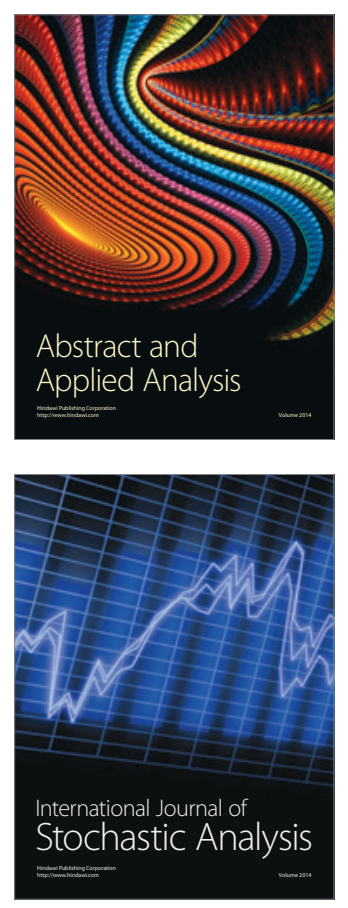

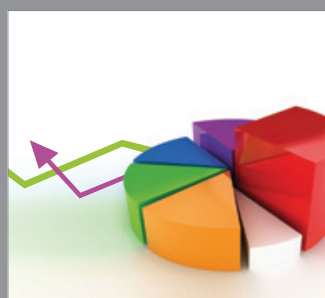

ournal of

Probability and Statistics

Promensencen
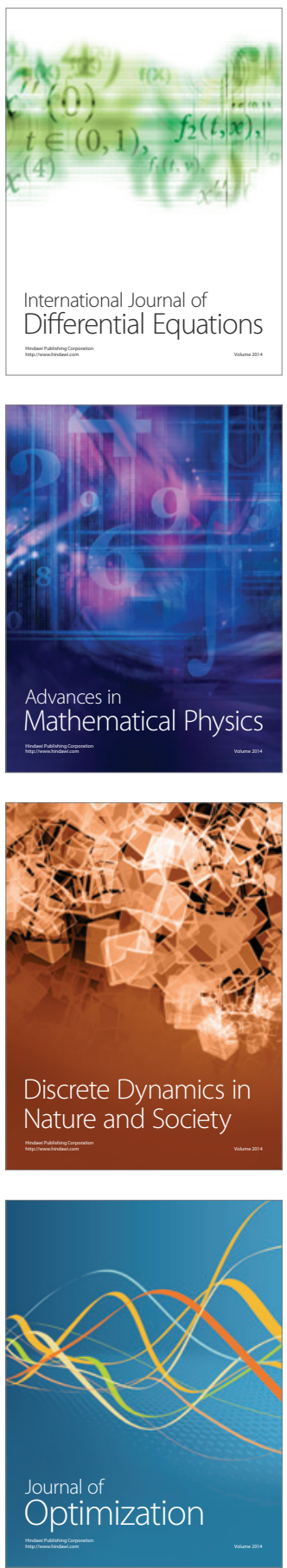